\title{
Optimal timing of percutaneous transhepatic gallbladder drainage and subsequent laparoscopic cholecystectomy according to the severity of acute cholecystitis
}

\author{
Jung Suk Lee, Seung Jae Lee, In Seok Choi, Ju Ik Moon
}

Department of Surgery, Konyang University Hospital, Konyang University College of Medicine, Daejeon, Korea

\begin{abstract}
Backgrounds/Aims: The optimal timing of percutaneous transhepatic gallbladder drainage (PTGBD) and subsequent laparoscopic cholecystectomy (LC) according to the severity of acute cholecystitis (AC) has not been established yet.

Methods: This single-center, retrospective study included 695 patients with grade I or II AC without common bile duct stones who underwent PTGBD and subsequent LC between January 2010 and December 2019. Difficult surgery (DS) (open conversion, subtotal cholecystectomy, adjacent organ injury, transfusion, operation time $\geq 90$ minutes, or estimated blood loss $\geq 100 \mathrm{~mL}$ ) and poor postoperative outcome (PPO) (postoperative hospital stay $\geq 7$ days or Clavien-Dindo grade $\geq$ II postoperative complication) were defined to comprehensively evaluate intraoperative and postoperative outcomes, respectively.

Results: Of 695 patients, 403 had grade I AC and 292 had grade II AC. According to the receiver operating characteristic curve and multivariate logistic regression analyses, an interval from symptom onset to PTGBD of $>3.5$ days and an interval from PTGBD to LC of $>7.5$ days were significant predictors of DS and PPO, respectively, in grade I AC. In grade II AC, the timing of PTGBD and subsequent LC were not statistically related to DS or PPO.

Conclusions: In grade I AC, performing PTGBD within 3.5 days after symptom onset can reduce surgical difficulties and subsequently performing LC within 7.5 days after PTGBD can improve postoperative outcomes. In grade II AC, early PTGBD cannot improve the surgical difficulty. In addition, the timing of subsequent LC is not correlated with surgical difficulties or postoperative outcomes.
\end{abstract}

Key Words: Acute cholecystitis; Laparoscopic cholecystectomy; Gallbladder; Drainage

\section{INTRODUCTION}

Acute cholecystitis (AC) is a common biliary disease for which laparoscopic cholecystectomy (LC) is the best treatment option [1]. Percutaneous transhepatic gallbladder drainage (PTGBD) was first reported in 1980 [2]. Since then, it has been used as an alternative treatment to avoid urgent sur-

Received: September 8, 2021, Revised: October 27, 2021

Accepted: October 29, 2021

Corresponding author: Seung Jae Lee

Department of Surgery, Konyang University Hospital, Konyang University College of Medicine, 158 Gwanjeodong-ro, Seo-gu, Daejeon 35365, Korea Tel: +82-42-600-9859, Fax: +82-42-543-8956, E-mail: leesj54gs@naver.com ORCID: https://orcid.org/0000-0002-3302-6624

Copyright (C) The Korean Association of Hepato-Biliary-Pancreatic Surger gery in high-risk or elderly patients [3]. Considering the high recurrence rate of $\mathrm{AC}$, subsequent $\mathrm{LC}$ is the only definitive treatment. Severity grading of AC according to the Tokyo Guidelines 2018 (TG 18) is significantly associated with intraoperative findings and postoperative outcomes [4]. Even in patients with AC undergoing PTGBD and subsequent LC, the difficulty of surgery and the postoperative complication rate may vary depending on the severity grading of the disease. When performing LC in patients with AC, main concerns are technical difficulties and postoperative complications associated with acute inflammation, adhesion, and severe fibrosis [5]. These factors may vary depending on whether and when PTGBD catheter is inserted. Therefore, it is essential to determine the optimal timing of PTGBD and subsequent LC. Additionally, the change after PTGBD may differ depending on the initial severity grading. However, studies on the optimal interval from the onset of symptoms to PTGBD and from PTGBD to $\mathrm{LC}$ according to the severity grading of AC are limited. Thus, 
the aim of the current study was to determine the relationship among surgical difficulties, postoperative outcomes, and the timing of PTGBD and subsequent LC.

\section{MATERIALS AND METHODS}

\section{Patients and data definitions}

Patients with AC who underwent subsequent LC after PTGBD at Konyang University Hospital between January 2010 and December 2019 were considered for inclusion. Patients with grade III AC or common bile duct (CBD) stones were excluded. A total of 695 patients were included in the study. The diagnosis and severity grading of AC were based on TG 18 [4].

This study was approved by the Institutional Review Board of Konyang University Hospital (approval no. 2021-08-016). The requirement for informed consent was waived owing to its retrospective study design.

In principle, patients with $\mathrm{AC}$ who cannot fit for immediate surgery due to the high risk of surgery at the time of presentation are indicated for PTGBD in our institution. Details differ depending on the grade of AC. PTGBD was performed in all patients with grade II or III AC if immediate LC was not possible for any reason. Patients with grade I AC were not treated with PTGBD at the time of presentation. However, PTGBD was performed during preparation for surgery if the clinical course worsened despite medical treatment. Other indications for PTGBD in grade I AC were jaundice (total bilirubin $\geq 2 \mathrm{mg} / \mathrm{dL}$ ) without CBD problems and impacted cystic duct stones.

The following patient characteristics were analyzed: age at surgery, sex, body mass index (BMI), American Society of Anesthesiologists physical status (ASA-PS) classification, history of previous abdominal surgery, onset of symptoms, method of LC, interval between PTGBD and LC, initial laboratory findings, intraoperative findings, and postoperative outcomes. The general condition and physical fitness of each patient were evaluated using the ASA-PS classification [6]. Information on the onset of symptoms such as fever, abdominal pain, and dyspepsia were obtained from patients' medical records. The method of LC was classified into single-incision LC and conventional multiport LC. The interval between PTGBD and LC was defined as the number of days from PTGBD catheter insertion to the subsequent LC surgery. Subtotal cholecystectomy was defined as making an incision in the gallbladder, aspirating the contents, and removing as much of the gallbladder wall as possible, with the aim of treating the stump instead of removing the entire gallbladder [7]. Adjacent organ injury was defined as unintended damage (requiring repair) to organs other than the gallbladder, such as the bile duct, duodenum, small bowel, and colon. The operation time was calculated as the time from skin incision to skin closure. Blood loss estimates were obtained from surgical records. Postoperative complications were graded according to the Clavien-Dindo classification [8]. Postoperative hospital stay was defined as the number of days of hospitalization after LC. Incisional hernia was defined as a hernia at the umbilical incision site postoperatively confirmed by physical examination and imaging studies.

\section{Definitions of difficult surgery and poor postoperative outcomes}

We comprehensively evaluated surgical outcomes by dividing them into intraoperative and postoperative factors. We defined difficult surgery (DS) as the occurrence of one of the following intraoperative outcomes: open conversion, subtotal cholecystectomy, adjacent organ injury during surgery, intraoperative transfusion, operation time $\geq 90 \mathrm{~min}$, and estimated blood loss $(\mathrm{EBL}) \geq 100 \mathrm{~mL}$. We defined poor postoperative outcome (PPO) as a postoperative hospital stay of $\geq 7$ days or a Clavien-Dindo $\geq$ grade II postoperative complication.

\section{Statistical analysis}

Continuous variables are expressed as mean and standard deviation. They were compared using Student's t-test or analysis of variance. Categorical variables are presented as counts and percentages. They were compared using the chi-squared test. Multivariate analyses of significant factors identified in the univariate analyses were performed using a logistic regression model. We also conducted receiver operating characteristic curve analyses of the relationship between the interval from the onset of symptoms to PTGBD and from PTGBD to LC and surgical outcomes. The cutoff value was calculated according to the Youden index. All tests were two-sided and $p$-values < 0.05 were considered statistically significant. All statistical analyses were performed using IBM SPSS version 22 (IBM Corp., Armonk, NY, USA).

\section{RESULTS}

\section{Study cohort}

Demographics and surgical outcomes of all patients according to the severity grading of AC are shown in Table 1. Among the 695 included patients, 403 were classified as having grade I AC and 292 were classified as having grade II AC. Patients with grade II AC were older (64.7 vs. 67.1 years; $p=0.031)$ than those with grade I AC. The proportion of patients with ASA$P S \geq 3$ was higher in the grade II AC group than in the grade I AC group, showing a marginal significance between the two (31.1\% vs. $38.0 \%$; $p=0.064$ ). The type of surgical method was not significantly different between the two groups (single-incision LC: $16.6 \%$ vs. $15.1 \% ; p=0.580$ ). The mean interval from PTGBD to LC was not significantly different either (6.0 vs. 6.3 days; $p=0.435)$. Subtotal cholecystectomy $(0.2 \%$ vs. $1.0 \% ; p=$ $0.180)$, adjacent organ injury during surgery $(1.2 \%$ vs. $3.1 \% ; p=$ $0.088)$, and intraoperative transfusion $(0.0 \%$ vs. $0.7 \% ; p=0.096)$ were more frequent in grade II AC than in grade I AC, although these differences were not statistically significant. The open conversion rate was significantly higher in patients with 
Table 1. Patients' demographics and surgical outcomes according to the severity of acute cholecystitis

\begin{tabular}{|c|c|c|c|c|}
\hline Variable & Total $(n=695)$ & Grade I $(n=403)$ & Grade II $(n=292)$ & $p$-value \\
\hline Female & $285(41.0)$ & $171(42.4)$ & $114(39.0)$ & \\
\hline Age (yr) & $65.7 \pm 14.6$ & $64.7 \pm 14.6$ & $67.1 \pm 14.4$ & 0.031 \\
\hline BMI $\left(\mathrm{kg} / \mathrm{m}^{2}\right)$ & $24.7 \pm 3.5$ & $24.8 \pm 3.8$ & $24.5 \pm 3.4$ & 0.421 \\
\hline ASA-PS classification & & & & 0.064 \\
\hline$\geq 3$ & $237(34.1)$ & $126(31.3)$ & $111(38.0)$ & \\
\hline Previous abdominal surgery & $109(15.7)$ & $67(16.6)$ & $42(14.4)$ & 0.422 \\
\hline Method of surgery & & & & 0.580 \\
\hline Single-incision LC & $111(16.0)$ & $67(16.6)$ & $44(15.1)$ & \\
\hline Conventional multiport LC & $584(84.0)$ & $336(83.4)$ & $248(84.9)$ & \\
\hline Initial platelet count $\left(10^{3} / \mathrm{mm}^{3}\right)$ & $221.3 \pm 74.6$ & $217.9 \pm 64.1$ & $226.1 \pm 86.8$ & 0.153 \\
\hline Initial PT (INR) & $1.13 \pm 0.14$ & $1.09 \pm 0.11$ & $1.18 \pm 0.16$ & $<0.001$ \\
\hline Initial creatinine level (mg/dL) & $1.01 \pm 0.96$ & $0.99 \pm 0.82$ & $1.04 \pm 1.13$ & 0.546 \\
\hline Initial total bilirubin level (mg/dL) & $1.58 \pm 1.42$ & $1.48 \pm 1.36$ & $1.72 \pm 1.49$ & 0.031 \\
\hline Initial AST level (IU/L) & $94.9 \pm 228.0$ & $94.7 \pm 194.8$ & $95.1 \pm 267.5$ & 0.983 \\
\hline Initial ALT level (IU/L) & $69.0 \pm 132.8$ & $74.1 \pm 140.4$ & $61.8 \pm 121.2$ & 0.226 \\
\hline Subtotal cholecystectomy & $4(0.6)$ & $1(0.2)$ & $3(1.0)$ & 0.180 \\
\hline Open conversion & $11(1.6)$ & $2(0.5)$ & $9(3.1)$ & 0.007 \\
\hline Adjacent organ injury & $14(2.0)$ & $5(1.2)$ & $9(3.1)$ & 0.088 \\
\hline Duodenum & $2(0.3)$ & $1(0.2)$ & $1(0.3)$ & \\
\hline Drain insertion & $184(26.5)$ & $70(17.4)$ & $114(39.0)$ & $<0.001$ \\
\hline Difficult surgery & $121(17.4)$ & $52(12.9)$ & $69(23.6)$ & $<0.001$ \\
\hline Postoperative hospital stay (day) & $3.9 \pm 5.0$ & $3.5 \pm 4.3$ & $4.5 \pm 5.8$ & 0.009 \\
\hline Total hospital stay (day) & $10.5 \pm 7.1$ & $10.0 \pm 6.8$ & $11.1 \pm 7.5$ & 0.062 \\
\hline Postoperative overall complication & $78(11.2)$ & $37(9.2)$ & $41(14.0)$ & 0.045 \\
\hline Postoperative complication $\geq$ grade $\| \mathrm{CDC}$ & $77(11.1)$ & $37(9.2)$ & $40(13.7)$ & 0.061 \\
\hline Postoperative complication $\geq$ grade III CDC & $32(4.6)$ & $13(3.2)$ & $19(6.5)$ & 0.042 \\
\hline Mortality at 30 days & $4(0.6)$ & $2(0.5)$ & $2(0.7)$ & 0.746 \\
\hline Poor postoperative outcomes & $101(14.5)$ & $46(11.4)$ & $55(18.8)$ & 0.006 \\
\hline Postoperative incisional hernia & $4(0.6)$ & $2(0.5)$ & $2(0.7)$ & 0.746 \\
\hline Acute cholecystitis in pathology & $352(50.6)$ & $181(44.9)$ & $171(58.6)$ & $<0.001$ \\
\hline
\end{tabular}

Values are presented as number (\%) or mean \pm standard deviation.

BMI, body mass index; ASA-PS, American Society of Anesthesiologists physical status; LC, laparoscopic cholecystectomy; PTGBD, percutaneous transhepatic gallbladder drainage; WBC, white blood cell; PT, prothrombin time; INR, international normalized ratio; AST, aspartate aminotransferase; ALT, alanine aminotransferase; CDC, Clavien-Dindo classification. 
grade II AC ( $3.1 \%$ vs. $0.5 \% ; p=0.007)$. The operation time $(68.2$ min vs. $57.1 \mathrm{~min} ; p<0.001)$ and postoperative hospital stay $(4.5$ days vs. 3.5 days; $p=0.009)$ were significantly longer and EBL (33.1 mL vs. $22.7 \mathrm{~mL}, p=0.012$ ) was significantly higher in patients with grade II AC than in those with grade I AC. Postoperative overall complications $(14.0 \%$ vs. $9.2 \% ; p=0.045)$ also more frequently occurred in patients with grade II AC. The incisional hernia incidence $(0.5 \%$ vs. $0.7 \% ; p=0.746)$ was similar between the two groups. There were four mortalities, two $(0.5 \%)$ in grade I AC and two (0.7\%) in grade II AC. All four patients with age of 75 years or older died of pneumonia after LC. They were not included in the DS group.

\section{Patients with grade I AC}

Results of receiver operating characteristic curve analyses for DS and PPO are shown in Fig. 1. The area under the curve (AUC) for the relationship between DS and the interval from symptom onset to PTGBD was 0.589 (95\% confidence interval [CI], 0.502-0.676; $p=0.038)$. The cutoff was calculated to be 3.5 days. The AUC for the relationship between DS and the interval from PTGBD to LC was 0.473 (95\% CI, 0.390-0.556; $p=0.528)$. The AUC for the relationship between PPO and the interval from symptom onset to PTGBD was 0.487 (95\% CI, $0.394-0.579 ; p=0.769)$. The AUC for the relationship between PPO and the interval from PTGBD to LC was 0.622 (95\% CI, $0.530-0.714 ; p=0.007)$. The cutoff was calculated as 7.5 days.

In univariate analysis for DS, BMI $\geq 30 \mathrm{~kg} / \mathrm{m}^{2}$ and interval from symptom onset to PTGBD > 3.5 days were statistically significant predictors of DS. In a multivariate logistic regression model that included these significant factors identified in the univariate analyses, $\mathrm{BMI} \geq 30 \mathrm{~kg} / \mathrm{m}^{2}$ and interval from symptom onset to PTGBD > 3.5 days were also statistically significant predictors of DS (Table 2). In univariate analysis for $\mathrm{PPO}$, age $\geq 70$ years, $\mathrm{BMI}<20 \mathrm{~kg} / \mathrm{m}^{2}$, ASA-PS $\geq 3$, interval from PTGBD to LC $>7.5$ days, and DS were statistically significant predictors of PPO. In multivariate analysis, ASA-PS $\geq 3$, interval from PTGBD to LC of $>7.5$ days, and DS were statistically significant predictors for PPO (Table 3).
A

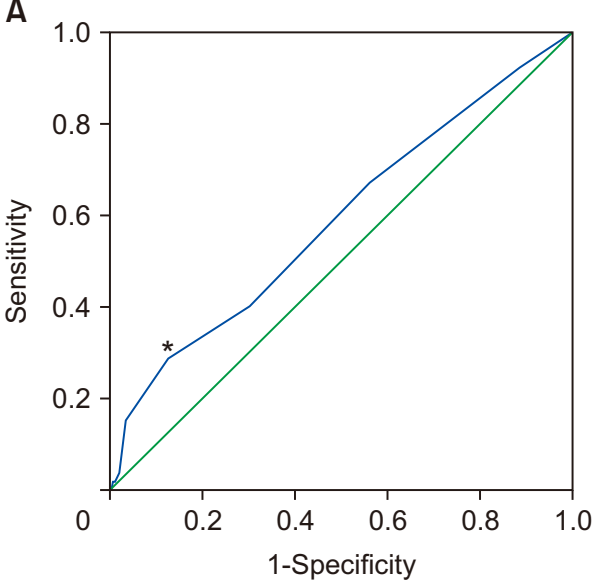

AUC $=0.589, \mathrm{Cl}=0.502-0.676, p=0.038$

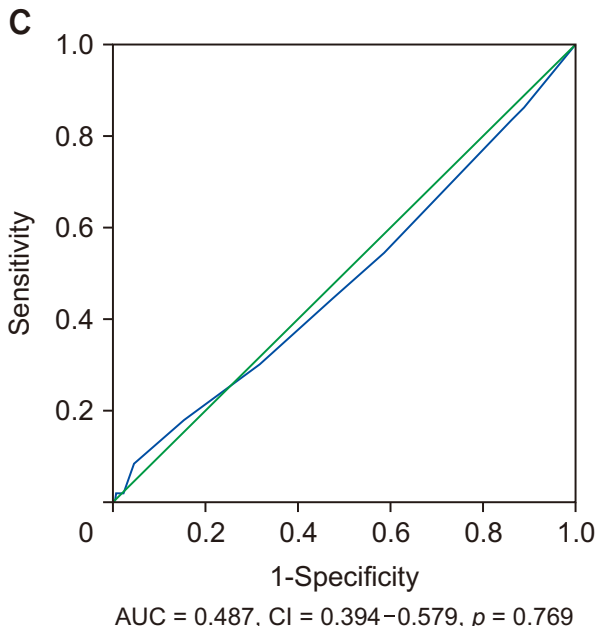

B

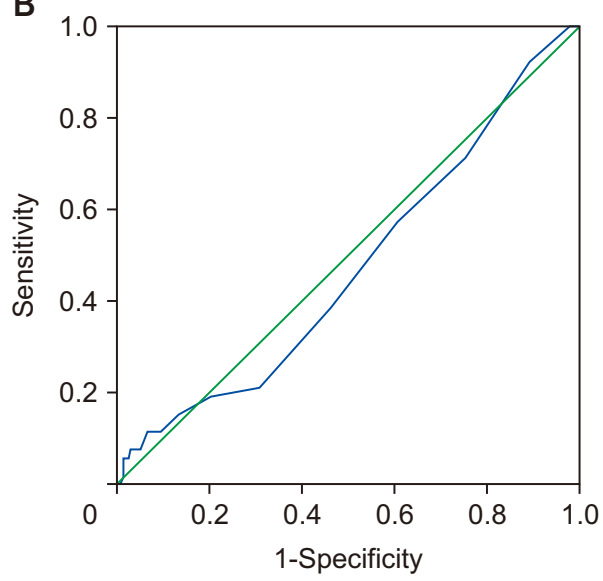

AUC $=0.473, \mathrm{Cl}=0.390-0.556, p=0.528$

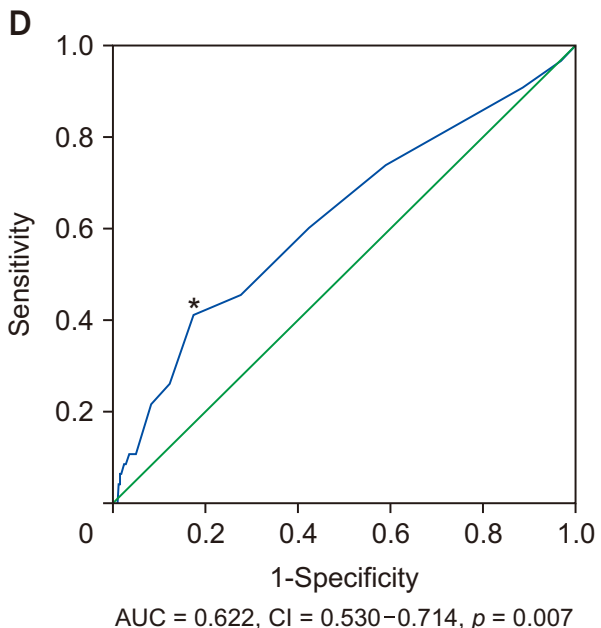

Fig. 1. ROC curve analysis in patients with grade I acute cholecystitis. (A) ROC curves between difficult surgery and interval from symptom onset to PTGBD. The AUC is 0.589 . *The cutoff is calculated as 3.5 days. (B) ROC curves between difficult surgery and interval from PTGBD to LC. The AUC is 0.473 . (C) ROC curves between poor postoperative outcome and interval from symptom onset to PTGBD. The AUC is 0.487. (D) ROC curves between poor postoperative outcome and interval from PTGBD to LC. The AUC is 0.622 . *The cutoff is calculated as 7.5 days. AUC, area under the curve; $\mathrm{Cl}$, confidence interval; LC, laparoscopic cholecystectomy; PTGBD, percutaneous transhepatic gallbladder drainage; ROC, receiver operating characteristic. 
Table 2. Univariate and multivariate analyses of risk factors for difficult surgery in patients with grade I acute cholecystitis

\begin{tabular}{|c|c|c|c|c|}
\hline Factor & \multicolumn{2}{|c|}{ Univariate analysis } & \multicolumn{2}{|c|}{ Multivariate analysis } \\
\hline \multicolumn{5}{|l|}{ Sex } \\
\hline Male & $1.779(0.952-3.326)$ & 0.071 & & \\
\hline \multicolumn{5}{|l|}{ Age } \\
\hline \multicolumn{5}{|l|}{ Body mass index } \\
\hline$<30 \mathrm{~kg} / \mathrm{m}^{2}$ & 1 (reference) & - & 1 (reference) & - \\
\hline$\geq 30 \mathrm{~kg} / \mathrm{m}^{2}$ & $2.719(1.141-6.478)$ & 0.024 & $2.734(1.130-6.615)$ & 0.026 \\
\hline \multicolumn{5}{|l|}{ ASA-PS classification } \\
\hline$<3$ & 1 (reference) & - & & \\
\hline Yes & $1.833(0.918-3.660)$ & 0.086 & & \\
\hline \multicolumn{5}{|c|}{ Interval from symptom onset to PTGBD } \\
\hline$\leq 3.5$ days & 1 (reference) & - & 1 (reference) & - \\
\hline$>3.5$ days & $2.757(1.401-5.423)$ & 0.003 & $2.766(1.397-5.476)$ & 0.004 \\
\hline \multicolumn{5}{|l|}{ Initial total bilirubin } \\
\hline$<2 \mathrm{mg} / \mathrm{dL}$ & 1 (reference) & - & & \\
\hline$\geq 2 \mathrm{mg} / \mathrm{dL}$ & $1.580(0.780-3.200)$ & 0.204 & & \\
\hline
\end{tabular}

OR, odds ratio; Cl, confidence interval; ASA-PS, American Society of Anesthesiologists physical status; PTGBD, percutaneous transhepatic gallbladder drainage.

\section{Patients with grade II AC}

Results of receiver operating characteristic curve analyses for DS and PPO are shown in Fig. 2. The AUC for the relationship between DS and the interval from symptom onset to PTGBD was 0.437 (95\% CI, 0.356-0.518; $p=0.114$ ). The AUC for the relationship between DS and the interval from PTGBD to LC was 0.493 (95\% CI, 0.418-0.569; $p=0.868$ ). The AUC for the relationship between PPO and the interval from symptom onset to PTGBD was 0.376 (95\% CI, $0.291-0.460 ; p=0.004)$. The cutoff was calculated as 2.5 days. The AUC for the relationship between PPO and the interval from PTGBD to LC was 0.560 (95\% CI, 0.471-0.650; $p=0.164$ ).

In univariate analysis, age $\geq 70$ years, BMI $<20 \mathrm{~kg} / \mathrm{m}^{2}$, ASA$\mathrm{PS} \geq 3$, interval from symptom onset to $\mathrm{PTGBD}<2.5$ days, and DS were statistically significant predictors of PPO. In multivariate analysis, ASA-PS $\geq 3$ and DS were statistically significant predictors of PPO. However, interval from symptom onset to PTGBD $<2.5$ days was not a statistically significant predictor of PPO in multivariate analysis (Table 4).

\section{DISCUSSION}

PTGBD is an alternative treatment that is used to avoid urgent surgery in high-risk patients with AC. Subsequent LC after PTGBD is considered the definitive treatment. Several observational studies have inconsistently reported the optimal timing of subsequent LC after PTGBD [9-12]. Another observational study has reported the ideal timing of PTGBD after the onset of AC [13]. However, no study has investigated the optimal timing of PTGBD or subsequent LC according to the severity of AC. The present study is meaningful as it is the first to report the optimal timing of PTGBD and subsequent LC according to the severity of AC.

Grade III AC is associated with organ or system dysfunction. It is not an indication for straightforward LC. According to TG 18 , appropriate organ support with initial medical treatment is recommended for grade III AC. LC should be chosen after a careful consideration of the patient's background, including complications and comorbidities [1]. Therefore, even if LC is selected, it is difficult to arbitrarily control the timing of surgery. Therefore, patients with grade III AC were excluded from the present study.

Several factors can be used for the evaluation of surgical outcomes. They can be divided into intraoperative factors (such as operation time and EBL) and postoperative factors (such as postoperative complications and length of hospital stay). Although these factors can influence each other, they sometimes have independent effects. It is difficult to evaluate each of these 
Table 3. Univariate and multivariate analyses of risk factors for poor postoperative outcomes in patients with grade I acute cholecystitis

\begin{tabular}{|c|c|c|c|c|}
\hline Factor & \multicolumn{2}{|c|}{ Univariate analysis } & \multicolumn{2}{|c|}{ Multivariate analysis } \\
\hline \multicolumn{5}{|l|}{ Sex } \\
\hline Male & $1.054(0.565-1.965)$ & 0.869 & & \\
\hline \multicolumn{5}{|l|}{ Age } \\
\hline \multicolumn{5}{|l|}{ Body mass index } \\
\hline$\geq 20 \mathrm{~kg} / \mathrm{m}^{2}$ & 1 (reference) & - & 1 (reference) & - \\
\hline$<20 \mathrm{~kg} / \mathrm{m}^{2}$ & $2.606(1.050-6.467)$ & 0.039 & $2.168(0.818-5.749)$ & 0.120 \\
\hline \multicolumn{5}{|l|}{ ASA-PS classification } \\
\hline$<3$ & 1 (reference) & - & 1 (reference) & - \\
\hline Yes & $0.728(0.296-1.792)$ & 0.490 & & \\
\hline \multicolumn{5}{|c|}{ Interval from PTGBD to LC } \\
\hline$\leq 7.5$ days & 1 (reference) & - & 1 (reference) & - \\
\hline$>7.5$ days & $3.284(1.720-6.271)$ & $<0.001$ & $2.672(1.342-5.321)$ & 0.005 \\
\hline \multicolumn{5}{|l|}{ Initial total bilirubin } \\
\hline$<2 \mathrm{mg} / \mathrm{dL}$ & 1 (reference) & - & & \\
\hline$\geq 2 \mathrm{mg} / \mathrm{dL}$ & $0.714(0.290-1.757)$ & 0.463 & & \\
\hline \multicolumn{5}{|l|}{ Difficult surgery } \\
\hline No & 1 (reference) & - & 1 (reference) & - \\
\hline Yes & $2.797(1.340-5.837)$ & 0.006 & $3.370(1.534-7.406)$ & 0.002 \\
\hline
\end{tabular}

OR, odds ratio; Cl, confidence interval; ASA-PS, American Society of Anesthesiologists physical status; PTGBD, percutaneous transhepatic gallbladder drainage; LC, laparoscopic cholecystectomy.

various factors in one study to determine the optimal timing of a procedure. Thus, it is necessary to evaluate them in an integrated manner. Therefore, we defined DS and PPO for a comprehensive evaluation of surgical outcomes.

Postoperative outcomes can be clearly evaluated on the basis of postoperative complications and length of hospital stay. However, the evaluation of intraoperative factors is difficult to define. The most serious intraoperative complication during LC is damage to the bile duct, vessels, or adjacent organs. TG 18 recommends choosing bail-out procedures to prevent bile duct injury according to intraoperative findings. Bail-out procedures for difficult LC include open conversion, subtotal cholecystectomy, and the fundus-first technique [7]. Iwashita et al. [14] have reported that most surgeons consider extensive blood loss as an unstable situation. In addition, most previous studies have used operation time $[15,16]$ or open conversion rate $[17,18]$ as surrogate markers of surgical difficulty. Therefore, we defined DS as the occurrence of open conversion, subtotal cholecystectomy, adjacent organ injury during surgery, intraoperative transfusion, prolonged operation time, or large EBL.

Most previous observational studies on the optimal timing of subsequent LC recommended delayed surgery after PTGBD. Previous studies reported that better surgical outcomes were obtained when LC was performed at 5 days [9], 9 days [12], or between 7 and 26 days [11] after PTGBD. However, in the present study, patients with grade I AC who underwent subsequent LC within 7.5 days after PTGBD showed better postoperative outcomes. In multivariate analysis, an interval from PTGBD to LC of $>7.5$ days was statistically significant predictor of PPO. It was confirmed that early LC within 7.5 days after PTGBD had a greater effect on postoperative outcomes than age or BMI in patients with grade I AC regardless of the difficulty of surgery. In contrast, the timing of subsequent LC was not correlated with postoperative outcomes in patients with grade II AC. Our results also revealed that the interval from PTGBD to subsequent LC did not influence the difficulty of LC in grade I or grade II AC. Therefore, a long interval from PTGBD to subsequent LC is not recommended because it is associated with prolonged hospitalization, high medical costs, and poor quality of life.

Studies on the optimal timing of PTGBD for the treatment of AC are rare. Yamada et al. [13] have reported that the most important predictor of successful LC after PTGBD for AC is 
A

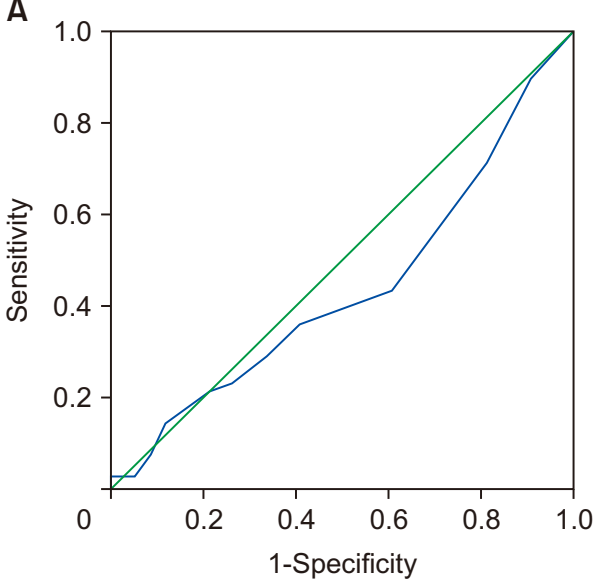

AUC $=0.437, \mathrm{Cl}=0.356-0.518, p=0.114$

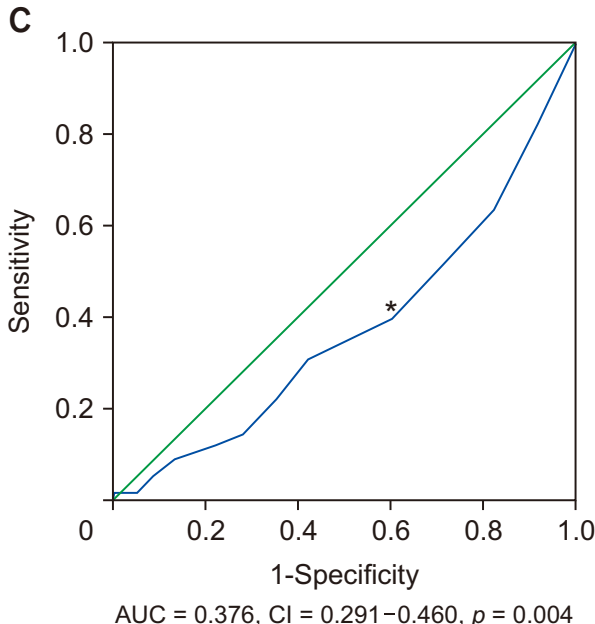

B

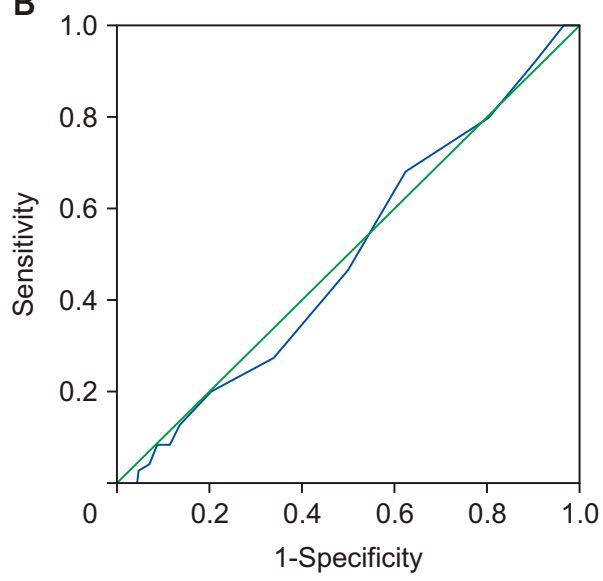

AUC $=0.493, \mathrm{Cl}=0.418-0.569, p=0.868$

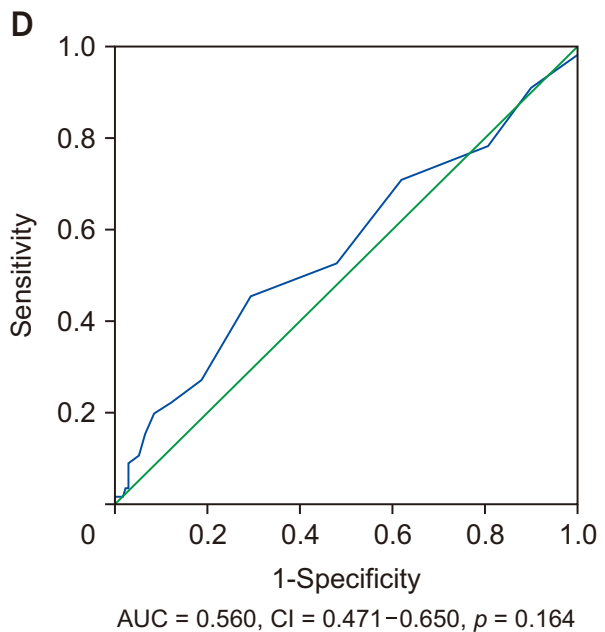

Fig. 2. ROC analysis in patients with grade II acute cholecystitis. (A) ROC curves between difficult surgery and interval from symptom onset to PTGBD. The AUC is 0.437 . (B) ROC curves between difficult surgery and interval from PTGBD to LC. The AUC is 0.493. (C) ROC curves between poor postoperative outcome and interval from symptom onset to PTGBD. The AUC is 0.376 . *The cutoff is calculated as 2.5 days. (D) ROC curves between poor postoperative outcome and interval from PTGBD to LC. The AUC is 0.560 . AUC, area under the curve; $\mathrm{Cl}$, confidence interval; LC, laparoscopic cholecystectomy; PTGBD, percutaneous transhepatic gallbladder drainage; ROC, receiver operating characteristic. an interval from symptom onset to PTGBD of $\leq 73.5$ hours. The authors believed that this was because PTGBD prevented the progression of AC. Similarly, in the present study, PTGBD within 3.5 days of symptom onset was reported as an important factor for successful LC in patients with grade I AC. However, our study found that the timing of PTGBD from symptom onset did not influence the postoperative outcomes of patients with grade II AC according to multivariate logistic regression analysis. Ábrahám et al. [19] have reported that after PTGBD, the level of gallbladder inflammation plays a decisive role in the course of $\mathrm{AC}$ (the higher the severity of $\mathrm{AC}$, the higher the mortality rate). Our results also revealed that early PTGBD did not improve surgical outcomes of patients with grade II AC. Therefore, for patients with grade II AC diagnosed within $72 \mathrm{~h}$, early LC without PTGBD is recommended if the patient's general condition allows surgery, as recommended by TG 18 [1].

In general, it is believed that intraoperative findings or events can affect postoperative outcomes. When analyzing predictive factors of PPO, DS was included in the present study. As a result of multivariate analysis, it was confirmed that DS was the most significant predictor for PPO in both grade I and II AC. Therefore, after complicated LC with difficulty, more detailed and meticulous postoperative management is necessary for preventing PPO.

Our study had several limitations. First, as this was a retrospective study, a selection bias might exist. In addition, the timing of symptom onset might not be accurate because this study relied on medical records. Second, this was the first study to define DS and PPO for a comprehensive evaluation of intraoperative and postoperative outcomes, respectively. However, the fact that we did not perform a detailed comparison of each surgical outcome factor could be viewed as a disadvantage. Finally, we excluded patients with CBD stones because the timing of LC might be affected by the treatment course of CBD stones. However, because many patients with AC have CBD stones, additional studies including patients with $\mathrm{CBD}$ stones are needed.

In conclusion, the optimal timing of PTGBD and that of subsequent LC can differ according to the severity of AC. In grade I AC, performing PTGBD within 3.5 days after symptom onset 
Jung Suk Lee, et al.

Table 4. Univariate and multivariate analyses of risk factors for poor postoperative outcomes in patients with grade II acute cholecystitis

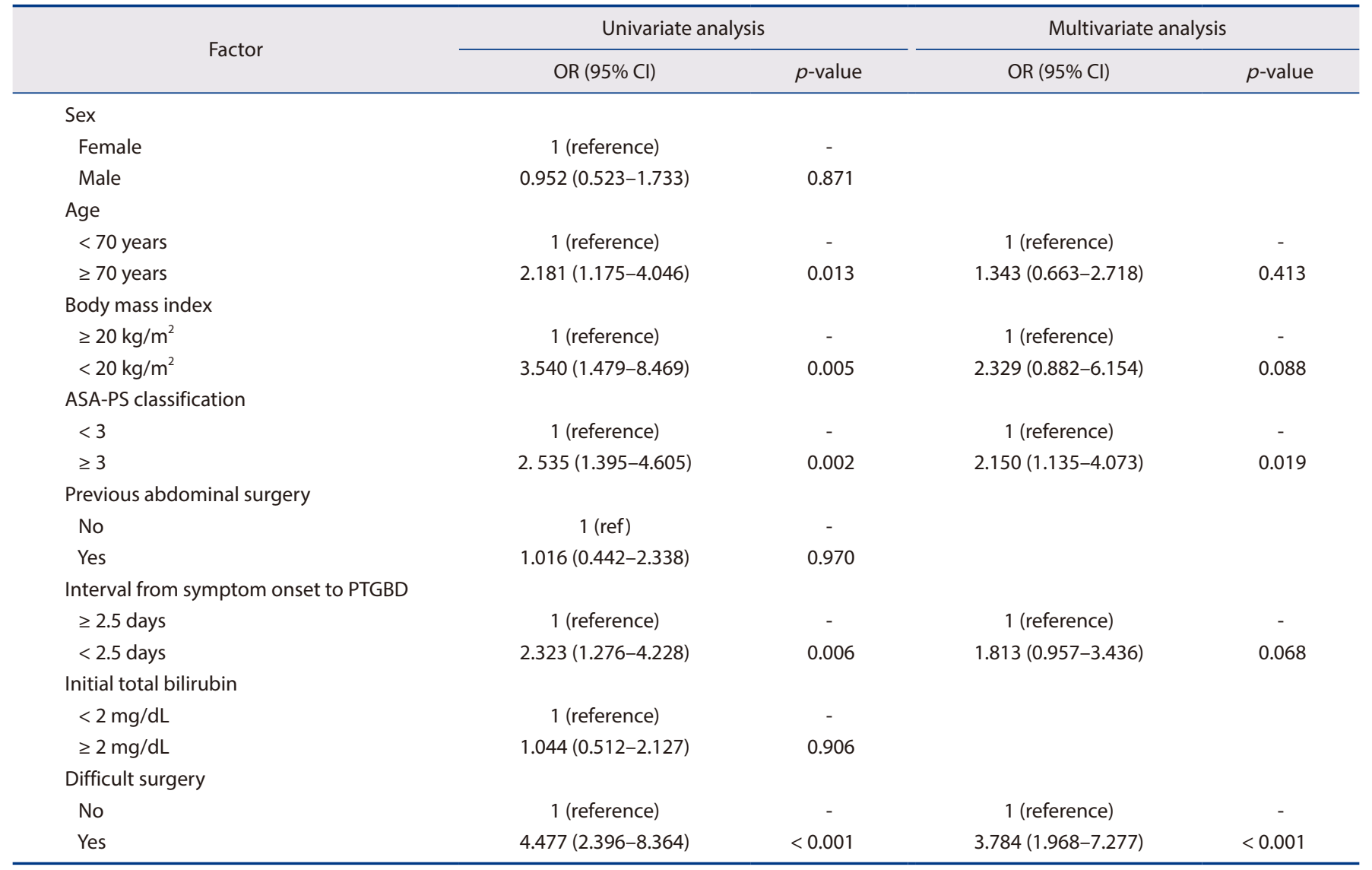

OR, odds ratio; $\mathrm{Cl}$, confidence interval; ASA-PS, American Society of Anesthesiologists physical status; PTGBD, percutaneous transhepatic gallbladder drainage.

can reduce surgical difficulties and subsequently performing LC within 7.5 days after PTGBD can improve postoperative outcomes. In grade II AC, early PTGBD does not improve the surgical difficulty. In addition, the timing of subsequent LC is not correlated with surgical difficulties or postoperative outcomes.

\section{FUNDING}

None.

\section{CONFLICT OF INTEREST}

No potential conflict of interest relevant to this article was reported.

\section{ORCID}

Jung Suk Lee, https://orcid.org/0000-0002-0583-9011

Seung Jae Lee, https://orcid.org/0000-0002-3302-6624

In Seok Choi, https://orcid.org/0000-0002-9656-3697

Ju Ik Moon, https://orcid.org/0000-0002-8120-5854

\section{AUTHOR CONTRIBUTIONS}

Conceptualization: ISC, JIM, SJL. Data curation: JSL, SJL. Methodology: JSL, SJL. Visualization: ISC, JIM, SJL. Writing original draft: JSL, SJL. Writing - review \& editing: ISC, SJL.

\section{REFERENCES}

1. Okamoto K, Suzuki K, Takada T, Strasberg SM, Asbun HJ, Endo I, et al. Tokyo Guidelines 2018: flowchart for the management of acute cholecystitis. J Hepatobiliary Pancreat Sci 2018;25:55-72.

2. Radder RW. Ultrasonically guided percutaneous catheter drainage for gallbladder empyema. Diagn Imaging 1980;49:330-333.

3. Horn T, Christensen SD, Kirkegård J, Larsen LP, Knudsen AR, Mortensen FV. Percutaneous cholecystostomy is an effective treatment option for acute calculous cholecystitis: a 10-year experience. HPB (Oxford) 2015;17:326-331.

4. Yokoe M, Hata J, Takada T, Strasberg SM, Asbun HJ, Wakabayashi G, et al. Tokyo Guidelines 2018: diagnostic criteria and severity grading of acute cholecystitis (with videos). J Hepatobiliary Pancreat Sci 2018;25:41-54 
5. Habib FA, Kolachalam RB, Khilnani R, Preventza O, Mittal VK. Role of laparoscopic cholecystectomy in the management of gangrenous cholecystitis. Am J Surg 2001;181:71-75.

6. Mayhew D, Mendonca V, Murthy BVS. A review of ASA physical status - historical perspectives and modern developments. Anaesthesia 2019;74:373-379.

7. Wakabayashi G, Iwashita Y, Hibi T, Takada T, Strasberg SM, Asbun HJ, et al. Tokyo Guidelines 2018: surgical management of acute cholecystitis: safe steps in laparoscopic cholecystectomy for acute cholecystitis (with videos). J Hepatobiliary Pancreat Sci 2018;25:73-86.

8. Clavien PA, Barkun J, de Oliveira ML, Vauthey JN, Dindo D, Schulick $\mathrm{RD}$, et al. The Clavien-Dindo classification of surgical complications: five-year experience. Ann Surg 2009;250:187-196.

9. Han IW, Jang JY, Kang MJ, Lee KB, Lee SE, Kim SW. Early versus delayed laparoscopic cholecystectomy after percutaneous transhepatic gallbladder drainage. J Hepatobiliary Pancreat Sci 2012;19:187-193.

10. Choi JW, Park SH, Choi SY, Kim HS, Kim TH. Comparison of clinical result between early laparoscopic cholecystectomy and delayed laparoscopic cholecystectomy after percutaneous transhepatic gallbladder drainage for patients with complicated acute cholecystitis. Korean J Hepatobiliary Pancreat Surg 2012;16:147-153.

11. Sakamoto T, Fujiogi M, Matsui H, Fushimi K, Yasunaga H. Timing of cholecystectomy after percutaneous transhepatic gallbladder drainage for acute cholecystitis: a nationwide inpatient database study. HPB (Oxford) 2020;22:920-926.

12. Inoue K, Ueno T, Nishina O, Douchi D, Shima K, Goto S, et al. Optimal timing of cholecystectomy after percutaneous gallbladder drainage for severe cholecystitis. BMC Gastroenterol 2017;17:71.
13. Yamada K, Yamashita Y, Yamada T, Takeno S, Noritomi T. Optimal timing for performing percutaneous transhepatic gallbladder drainage and subsequent cholecystectomy for better management of acute cholecystitis. J Hepatobiliary Pancreat Sci 2015;22:855-861.

14. Iwashita Y, Ohyama T, Honda G, Hibi T, Yoshida M, Miura F, et al. What are the appropriate indicators of surgical difficulty during laparoscopic cholecystectomy? Results from a Japan-Korea-Taiwan multinational survey. J Hepatobiliary Pancreat Sci 2016;23:533-547.

15. Schrenk P, Woisetschläger R, Rieger R, Wayand WU. A diagnostic score to predict the difficulty of a laparoscopic cholecystectomy from preoperative variables. Surg Endosc 1998;12:148-150.

16. Sakuramoto S, Sato S, Okuri T, Sato K, Hiki Y, Kakita A. Preoperative evaluation to predict technical difficulties of laparoscopic cholecystectomy on the basis of histological inflammation findings on resected gallbladder. Am J Surg 2000;179:114-121.

17. Simopoulos C, Botaitis S, Polychronidis A, Tripsianis G, Karayiannakis AJ. Risk factors for conversion of laparoscopic cholecystectomy to open cholecystectomy. Surg Endosc 2005;19:905-909.

18. Lipman JM, Claridge JA, Haridas M, Martin MD, Yao DC, Grimes $\mathrm{KL}$, et al. Preoperative findings predict conversion from laparoscopic to open cholecystectomy. Surgery 2007;142:556-563; discussion 563565.

19. Ábrahám S, Tóth I, Benkő R, Matuz M, Kovács G, Morvay Z, et al. Surgical outcome of percutaneous transhepatic gallbladder drainage in acute cholecystitis: ten years' experience at a tertiary care centre. Surg Endosc 2021. https://doi.org/10.1007/s00464-021-08573-0 [in press] 